\title{
A STUDY OF POSTERIOR CONDYLAR FORAMEN IN HUMAN SKELETAL IN CENTRAL GUJARAT REGION
}

\section{Hetal V. Vaishnani ${ }^{1}$, Kinjal Jethva *2, Priyanka Sharma ${ }^{3}$, A.R.Gandotra ${ }^{4}$, G.V.Shah ${ }^{5}$.}

${ }^{1}$ Associate Professor, Department of Anatomy, Smt. B.K.shah Medical Institute \& Research Centre, Sumandeep Vidyapeeth, Piparia, Vadodara, Gujarat.

${ }^{* 2}$ Assistant Professor, Department of Anatomy, Smt. B.K.shah Medical Institute \& Research Centre, Sumandeep Vidyapeeth, Piparia, Vadodara, Gujarat.

${ }^{3}$ Tutor, Department of Anatomy, Smt. B.K.shah Medical Institute \& Research Centre, Sumandeep Vidyapeeth, Piparia, Vadodara, Gujarat.

${ }^{4}$ Professor \& HOD, Department of Anatomy, Smt. B.K.shah Medical Institute \& Research Centre, Sumandeep Vidyapeeth, Piparia, Vadodara, Gujarat.

${ }^{5}$ Dean, \& Professor, Department of Anatomy, Smt. B.K.shah Medical Institute \& Research Centre, Sumandeep Vidyapeeth, Piparia, Vadodara, Gujarat.

\section{ABSTRACT}

Introduction: Posterior condylar foramen is the most constantly present emissary foramen in human skull. It is also considered as largest emissary foramen present in humans. Condylar emissary vein running along its path ensures patency of this passage

Aim: To assess normal location and variability in posterior condylar foramen

Materials and Methods: This study was conducted on 60 dry adult skulls gathered from the departments of anatomy, Smt.B.K.Shah Medial Institute \& Research Centre., to observe weather posterior condylar canal is located on one side or two sides of midline. Furthermore to study the morphologic anatomy of posterior condylar canal and its variations. Protocol of current study was the presence of complete condylar foramina behind occipital condyles as variation for the present study.

Results: Posterior condylar foramina were investigated in 60 human cadaveric skulls. Current research showed incidence of presence of posterior condylar foramina as $73.33 \%$. Amongst which we found bilaterally located posterior condylar foramina in 40\%, and Unilateral 33.33\% . Posterior Condylar foramen found absent in 26.66 $\%$ cadaveric Skulls.

Conclusion: The posterior condylar canal is one of the larger emissary channels in the skull. Research work on Condylar foramina provides fundamental information the clinicians, radiologists, anatomists and surgeons prior to operative procedures involving base of skull.

KEY WORDS: Emissary Vein, Posterior condylar Foramen, bilateral, unilateral, variations.

Address for Correspondence: Dr.Kinjal Jethva, Department of Anatomy, Smt.B.K.Shah Medical institute \& Research, Sumandeep Vidyapeeth, M - 9924870827 E-Mail: dr.hetal24@gmail.com

\begin{tabular}{|c|c|c|}
\hline \multicolumn{3}{|c|}{ Access this Article online } \\
\hline \multirow{2}{*}{$\begin{array}{l}\text { Quick Response code } \\
\text { Dol: } 10.16965 / \text { ijar.2017.460 }\end{array}$} & \multicolumn{2}{|c|}{$\begin{array}{l}\text { Web site: International Journal of Anatomy and Research } \\
\text { ISSN 2321-4287 } \\
\text { www.ijmhr.org/ijar.htm }\end{array}$} \\
\hline & $\begin{array}{l}\text { Received: } 25 \text { Jun } 2017 \\
\text { Peer Review: } 26 \text { Sep } 2017 \\
\text { Revised: None }\end{array}$ & $\begin{array}{l}\text { Accepted: } 08 \text { Nov } 2017 \\
\text { Published (O): } 01 \text { Dec } 2017 \\
\text { Published (P): } 01 \text { Dec } 2017\end{array}$ \\
\hline
\end{tabular}

\section{INTRODUCTION}

The condylar canal (or condyloid canal) is a canal in the condyloid fossa of the lateral parts of occipital bone behind the occipital condyle. 
Resection of the rectus capitus posterior major and minor muscles reveals the bony recess leading to the condylar canal, which is situated posterior and lateral to the occipital condyle. Through the condylar canal, the occipital emissary vein connects to the venous system including the suboccipital venous plexus, occipital sinus and sigmoid sinus [1].

In humans, the venous drainage of the posterior fossa has frequent anatomical variations. Most variations involving the lateral sinus in humans allow conservation of the IJV and VVS as their major outflow pathways. Only rarely, when the sigmoid sinus is absent or severely hypoplastic, may a petrosquamosal sinus, mastoid emissary or posterior condylar emissary vein represent the major or only drainage pathway of the transverse sinus [2,3].

Emissary condylar veins are divided into three groups as posterior, lateral and anterior condylar vein $[4,5]$. The posterior condylar vein usually took its origin from the superior bulb of the internal jugular vein. It courses along the posterior condylar vein canal, emerges from its foramen and drains into the deep cervical vein

Emissary veins are important clinically because infections from outside cranial cavity can spread to dural venous sinuses. For example infection can spread from mastoid to sigmoid sinus or from paranasal sinuses to cavernous sinus. These emissary veins provide an alternative venous drainage when internal jugular vein is blocked or tied [6]. Occipital condyles are convex surfaces covered with hyaline cartilage. They lie at front half of the foramen magnum. Their posterior poles are separated by the diameter of foramen magnum, but their anterior poles are much closure together. Behind the condyle is a shallow fossa floored by thin bone. This fossa shows perforation by posterior condylar canal. It (posterior condylar canal) carries vein from the sigmoid sinus to the suboccipital venous plexus [7].

The condylar canal (or condyloid canal) is a canal in the condyloid fossa of the lateral parts of occipital bone behind the occipital condyle. The posterior condylar canal is the largest emissary foramen of the posterior cranial fossa. It is apparent just posteroinferior to the jugular foramen and posterior to the hypoglossal canal
[8]. The Condylar canal gives way to a condylar emissary vein from sigmoid sinus to vertebral vein between the axis and atlas, in most cases 1 or between the superior bulb of the internal jugular vein and suboccipital venous plexus [9]. The posterior condylar foramen is located behind the condylar of the occipital bone.

Meningeal branches of the occipital artery also course through posterior condylar canal. This anatomical information is of vital significance to surgeons doing operative work on base of skull to avoid damage to the neurovascular structures $[10,11]$. The condylar veins can be used as access routes to hypoglossal dural arteriovenous fistulas. The surgical anatomy of posterior condylar foramina is of real importance during the management of clinical settings involving treatment of dural arteriovenous fistula.

\section{MATERIALS AND METHODS}

This study was conducted on 60 dry adult skulls gathered from the departments of anatomy, Smt.B.K.Shah Medial Institute \& Research Centre., to observe weather posterior condylar canal is located on one sided or two sides of midline. Furthermore to study the morphologic anatomy of posterior condylar canal and its variations. Protocol of current study was the presence of complete condylar foramina behind occipital condyles as variation for the present study. Methodology included passing a probe into the posterior condylar foramina to observe.

Skulls showing gross asymmetry or deformity particularly involving foramen magnum and occipital condyle region were rejected as unsuitable. Posterior condylar foramen is canal which is bent and it is sometimes very difficult to a ssess whether posterior condylar foramen is complete or not. When posterior condylar foramen was present only as pit or small blind canal, it was recorded as absent. Posterior condylar foramen was observed for its presence on one or both sides and whether it was absent on both sides.

\section{RESULTS}

Posterior condylar foramina were investigated in 60 human cadaveric skulls. Current research showed incidence of presence of posterior condylar foramina as $73.33 \%$. Amongst which we found bilaterally located posterior condylar 
foramina in $40 \%$, and Unilateral $33.33 \%$. Posterior Condylar foramen found absent in $26.66 \%$ cadaveric Skulls.

Fig. 1: Shows Present, Absent, Unilaterally (Right of Left Side) location of Condylar foramen.

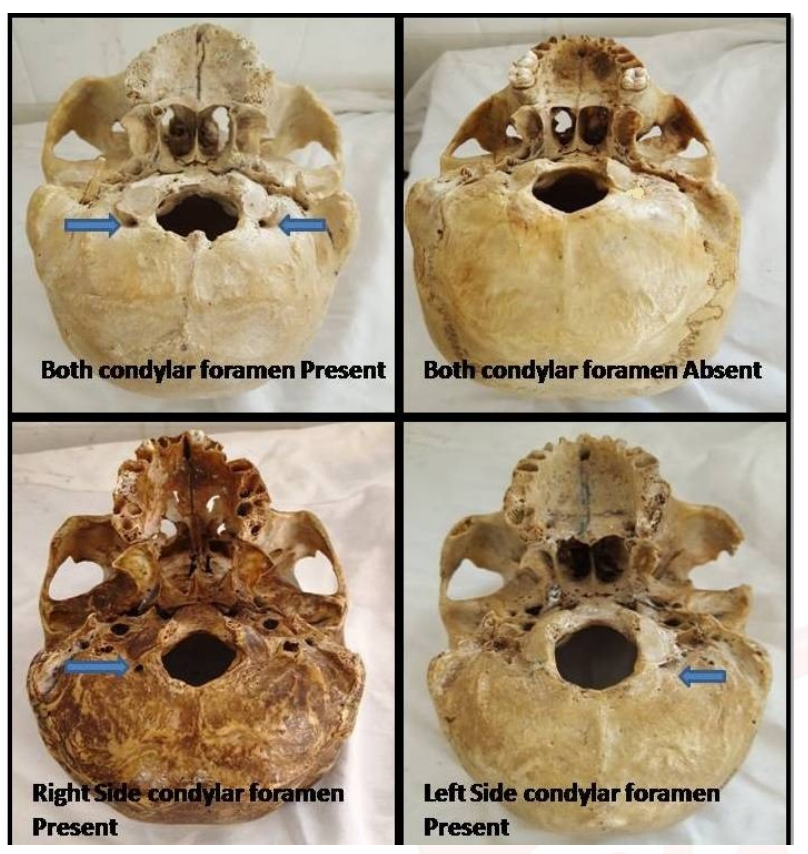

Table 1: No.of Posterior condylar foramina.

\begin{tabular}{|c|c|c|}
\hline Foramen & Present & Absent \\
\hline $\begin{array}{c}\text { Posterior Condylar } \\
\text { Forman }\end{array}$ & $44(73.33 \%)$ & $16(26.66 \%)$ \\
\hline
\end{tabular}

Table 2: Posterior Condylar Foramina.

\begin{tabular}{|c|c|c|c|}
\hline \multirow{2}{*}{ Foramen } & \multicolumn{2}{|c|}{ Unilateral } & \multirow{2}{*}{ Bilateral } \\
\cline { 2 - 3 } & Right Side & Left Side & \\
\hline $\begin{array}{c}\text { Posterior Condylar } \\
\text { Forman }\end{array}$ & $12(20 \%)$ & $08(13.33 \%)$ & $24(40 \%)$ \\
\hline
\end{tabular}

\section{DISCUSSION}

Correct identification of posterior condylar canal is important in computed tomography (CT) or magnetic resonance imaging (MRI) studies as posterior condylar canal can be wrongly interpreted as tumour of jugular fossa region or as lymph node which is enlarged. Sometimes large canal can be mistaken for anomalous blood vessel. Posterior condylar canal sometimes transmit ascending pharyngeal branch. Knowledge of posterior condylar canal is also important to surgeons operating in posterior cranial fossa region.

The posterior condylar canal forms a communication between the jugular foramen and the condylar fossa just posterior to the occipital condyles. It transmits an emissary vein which allows anastomosis of the jugular bulb or sigmoid sinus to the suboccipital venous plexus $[12,13]$. Variations are law of nature and significant variations have been observed in cerebral venous return into systemic veins 11. This has been a priority research topic of modern time in anatomical circles. This is pertinent to mention here that these anomalous foramina might be acting as additional source of drainage of the intracranial veins into extracranial veins.

Ginsberg et al observed the posterior condylar canal to be bilateral in $55.9 \%$ of his study work and was located unilaterally in $17.6 \%$ [14].

In a research work performed by Boyd, posterior condylar canal was observe the posterior condylar canal with an incidence of $77 \%$ unilaterally [10].

Galarza et al. in their study documented posterior condylar foramina of intrasinusal form in $24.6 \%$ of cases bilaterally, in which $17.8 \%$ were on the right side and $13.5 \%$ were found to be on the opposite location, whereas the type in which it present behind the sinus, the posterior condylar foramina was found in $1.2 \%$ of both sides and $1.2 \%$ observed on the right side [9].

Krause et al discovered that condylar canal was found on both sides in $21 \%$ and on one side only in 38\% [8]. Mian Ahmed et al found the incidence of posterior condylar foramina was $33 \%$, on two sides in $18 \%$, and on one side in $14 \%[15]$.

Usha Kothandaraman et al found that the incidence of posterior condylar foramina was 26\%: bilateral incidence was $16 \%$, whereas unilateral was $10 \%$ [16].

In our present study The posterior condylar foramina was present in $73.33 \%$ cases and absent in $26.66 \%$ of cases. And it was bilaterally in $40 \%$ skull and in unilaterally $33.33 \%$ (Right side $20 \%$ and Left side $13.33 \%$ ).

\section{CONCLUSION}

The posterior condylar canal is one of the larger emissary channels in the skull. Research work on Condylar foramina provides fundamental information the clinicians, radiologists, anatomists and surgeons prior to operative procedures involving base of skull. 
Anatomical variations of the posterior condylar foramina are important during the treatment of dural arteriovenous fistula.

\section{Conflicts of Interests: None}

\section{REFERENCES}

[1]. Standring Suzan. Gray's Anatomy: Anatomical Basis of Clinical Practice. 40th edition. Elsevier, Churchill Livingstone. London. 2008:432-433.

[2]. San Millán Ruíz D, Gailloud P, Rüfenacht DA, Delavelle J, Henry F, Fasel JH. The craniocervical venous system in relation to cerebral venous drainage. AJNR Am J Neuroradiol 2002;23:1500-8.

[3]. Giesemann AM, Goetz GF, Neuburger J, Lenarz T, Lanfermann H. Persistent petrosquamosal sinus: high incidence in cases of complete aplasia of the semicircular canals. Radiology 2011;259:825-33.

[4]. Reis CV, Deshmukh V, Zabramski JM, Crusius M, Desmukh P, Spetzler RF, et al. Anatomy of the mastoid emissary vein and venous system of the posterior neck region: neurosurgical implications. Neurosurgery 2007;61:193-200.

[5]. Jeevan DS, Anlsow P, Jayamohan J. Abnormal venous drainage in syndromic craniosynostosis and the role of CT venography. Childs Nerv Syst. 2008Dec;24(12):1413-20.

[6]. Standring Suzan. Gray's Anatomy: Anatomical Basis of Clinical Practice. 40th edition. Elsevier, Churchill Livingstone. London. 2008:432-433.

[7]. Last RJ. Anatomy Regional and Applied. 7th edition. ELBS. Churchill Livingstone, Edinburgh. 1984:561562.
[8]. Krause W.The posterior condylar canal.In Testut, L.\&Latarjet, A. Treaty of human anatomy.Barcelona, Salvat, 1988;1:152-8.

[9]. Coin MCG, Foramen M. In: Newton TH \& Potts DG. Radiology of the skull and brain: the skull. St. Louis, Mosby, 1971;1:275-86.

[10]. Boyd GI. The emissary foramina of the cranium in man and the anthropoids. J Anat. 1930;65(Pt 1): 10821.

[11]. Galarza M, Yun jong H, Merlo A. Chilean. J Anat. 1998;16(1):83-7.

[12]. Williams PL, Warwick R, Dyson M, Bannister LH, eds. Gray's anatomy, 37th ed. New York : Churchill Livingstone, 1989:286- 287

[13]. Haas LL. The posterior condylar fossa, foramen and canal, and the jugular foramen. Radiology 1957;69:546- 552

[14]. Ginsberg LE. The posterior condylar canal. AJNR Am J Neuroradiol. 1994;15(5):969-72.

[15]. Milan AA , Lal MK, Ibrahim K, Navab MK ; A Study of Morphology of Posterior Condylar Foramen and Its Anatomical Variations in Dry Pakistani Adult Skulls, P J M H S 2016;10(3):692-694.

[16]. Usha Kothandaraman, Sadhu Lokanadham, Posterior condylar foramen-anatomical variation, International Journal of Medical Science and Public Health, 2015;4(2):222-224.

How to cite this article:

Hetal V. Vaishnani, Kinjal Jethva, Priyanka Sharma, A.R.Gandotra, G.V.Shah. A STUDY OF POSTERIOR CONDYLAR FORAMEN IN HUMAN SKELETAL IN CENTRAL GUJARAT REGION. Int J Anat Res 2017;5(4.3):4736-4739. DOI: 10.16965/ijar.2017.460 